\title{
On the Minimal Displacement Problem of $\gamma$-Lipschitz Maps and $\gamma$-Lipschitz Retractions onto the Sphere
}

\author{
M. Väth
}

\begin{abstract}
We give a general construction in arbitrary normed spaces to produce fixedpoint free continuous maps with a large minimal displacement, contractions of the sphere, and retractions onto the sphere such that the corresponding maps have small measures of non-compactness.
\end{abstract}

Keywords: Condensing operator, measure of non-compactness, fixed point, minimal displacement, retraction

AMS subject classification: Primary 47H09, 47H10, secondary 46B20, 46B26, 47H12

\section{Introduction}

Let $X$ be a normed space, and let

$$
\begin{aligned}
B_{r}(X) & :=\{x \in X:\|x\| \leq r\} \\
S_{r}(X) & :=\{x \in X:\|x\|=r\} .
\end{aligned}
$$

It is well-known that the following statements are equivalent in $X$ :

1. There is a fixed-point free continuous map $F: B_{1}(X) \rightarrow B_{1}(X)$.

2. There is a homotopy $H: S_{1}(X) \times[0,1] \rightarrow S_{1}(X)$ which joins the identity with a constant map, i.e. $H(x, 0)=x$ and $H(x, 1) \equiv$ const for $x \in S_{1}(X)$.

3. There is a retraction of $B_{1}(X)$ onto $S_{1}(X)$, i.e. a continuous map $R: B_{1}(X) \rightarrow$ $S_{1}(X)$ with $R(x)=x$ on $S_{1}(X)$.

Indeed, if $F$ respectively $H$ are given, then $H$ respectively $R$ can be obtained by well-known constructions (which we recall later). Conversely, $-R$ is a fixed-point free map. If $X$ has finite dimensions, the above statements all fail in view of Brouwer's fixed point theorem. Conversely, if $X$ has infinite dimensions, the existence of a retraction of $B_{1}(X)$ onto $S_{1}(X)$ was first proved in [5], using the axiom of choice.

M. Väth: Univ. of Würzburg, Dept. Math., Am Hubland, D-97074 Würzburg vaeth@mathematik.uni-wuerzburg.de

This paper was written in the framework of a DFG Heisenberg scholarship. Financial support by the DFG is gratefully acknowledged. 
However, other constructions were found later, which require only the (countable) axiom of dependent choices: A very simple construction of a fixed-point free continuous map of $B_{1}(X)$ was given in [10]. This construction was later extended in many respects. Nowadays, it is known that (in each infinite-dimensional normed space) there exist Lipschitz maps with the above properties $[3,11,12]$.

Almost nothing is known about the best possible Lipschitz constant; all known constructions of the above maps have enormous Lipschitz constants (see [9] for a summary of known results in this direction; see also $[6,7]$ ). A well-known fixed point theorem on non-expanding maps implies that at least in uniformly convex spaces the Lipschitz constant of $F$ (and thus of $R$ ) must be larger than 1 (see, e.g., [13, 15] or [9]). It is actually known that the Lipschitz constant of $R$ must be at least 3 .

It turns out that the Lipschitz constant for $H$ and $R$ by the above mentioned constructions not only depends on the Lipschitz constant of $F$ but also on the minimal displacement

$$
\operatorname{disp}(F, M):=\inf \{\|F(x)-x\|: x \in M\} \quad(M \subseteq X)
$$

of $F$ on $M=B_{1}(X)$. Thus, one is interested not only in finding fixed-point free maps with a small Lipschitz constant but also with large $\operatorname{disp}\left(F, B_{1}(X)\right)$. This problem has been discussed in [14].

We are interested in a related question: What are the best possible constants such that $F, H, R$ are $\gamma$-Lipschitz where $\gamma$ denotes a measure of non-compactness?

Definition 1. For a set $M$ in a metric space, putting $\inf \emptyset=\infty$ and $\sup \emptyset=0$, we define:

1. The Kuratowski measure of non-compactness $\alpha(M)$ is the infimum of all $\varepsilon>0$ such that $M$ has a finite covering of sets with diameter at most $\varepsilon$.

2. The Hausdorff measure of non-compactness $\chi_{Y}(M)$ (with respect to a set $Y$ ) is the infimum of all $\varepsilon>0$ such that $M$ has a finite $\varepsilon$-net in $Y$.

3. The lattice measure of non-compactness $\beta(M)$ (in literature also called separation measure of non-compactness) is the supremum of all $\varepsilon>0$ such that $M$ contains a sequence $x_{n}$ with $d\left(x_{n}, x_{k}\right) \geq \varepsilon \quad(n \neq k)$.

The term "measure of non-compactness" is explained by the fact that the above measures vanish if and only if $M$ is precompact, i.e. if the completion of $M$ is compact (for the Hausdorff measure of non-compactness, assume here that $M \subseteq Y$ ). It is well-known and not hard to see that

$$
\chi_{Y}(M) \leq \chi_{Y_{0}}(M) \leq \beta(M) \leq \alpha(M) \leq 2 \chi_{Y}(M) \quad\left(M \subseteq Y_{0} \subseteq Y\right)
$$

It is also known that for subsets of a normed space $X$ the measures of non-compactness $\gamma \in\left\{\alpha, \beta, \chi_{X}\right\}$ are monotone, subadditive, homogeneous, and invariant under passage to the closed convex hull (see, e.g., [1,2]). A map $F: M \subseteq X \rightarrow X$ is called $\gamma$-Lipschitz, if there is some constant $L<\infty$ with

$$
\gamma(F(A)) \leq L \gamma(A) \quad(A \subseteq M)
$$


The name is explained by the fact that compact perturbations of Lipschitz maps are $\alpha$-Lipschitz (with at most the same constant $L$ ). It follows from Darbo's fixed point theorem [4] (and its extension of Sadovskiu [16]) that any fixed-point free continuous map $F: B_{1}(X) \rightarrow B_{1}(X)$ must satisfy $L \geq 1$ for $\gamma \in\left\{\alpha, \beta, \chi_{X}\right\}$ (if $X$ is a Banach space). In [17], we gave a construction of such a map which reaches the value $L=2$ in any normed space and the best possible value $L=1$ in a large class of spaces. The latter was formulated in [17] only for spaces with a "separable retraction property" as well as for Hilbert spaces and for "sufficiently large" subspaces of $l_{p}(S) \quad(1 \leq p \leq \infty)$, but an inspection of the proof shows that it works also for all normed spaces which contain an isometric copy of $c_{\mathrm{fin}, p}$ (the separable retraction property and containment of $c_{\text {fin, } p}$ will also play a special role in the current paper).

As in the Lipschitz case, the $\gamma$-Lipschitz constants for $H$ and $R$ depend also on $\operatorname{displ}\left(F, B_{1}(X)\right)$. Since $\operatorname{displ}\left(F, B_{1}(X)\right)=0$ in the construction from [17], this example cannot be used to construct, e.g., $\gamma$-Lipschitz retractions onto the unit sphere. This difficulty is of principal nature as was observed in [8]. It is not accidental that $\operatorname{displ}\left(F, B_{1}(X)\right)=0$ in the construction of [17]: If $L \geq 1$ is the $\gamma$-Lipschitz constant of $F$ for some $\gamma \in\left\{\alpha, \beta, \chi_{X}\right\}$ (in a Banach space $X$ ), then

$$
\operatorname{displ}\left(F, B_{1}(X)\right) \leq 1-\frac{1}{L}
$$

This was proved in [8] for the case $\gamma=\alpha$, but the same proof works also for $\gamma=\beta$ and $\gamma=\chi_{X}$.

We are thus interested in the construction of a continuous map $F: B_{1}(X) \rightarrow$ $B_{1}(X)$ with a small $\gamma$-Lipschitz constant such that $\operatorname{displ}\left(F, B_{1}(X)\right)$ is large.

In the space $X=C([0,1])$, there exist $\chi_{X}$-Lipschitz retractions onto $S_{1}(X)$ with any constant $L>1$ [18]. It is unknown whether the constant $L=1$ can be reached in some space. J. Wośko observed in [18] that this value cannot be reached for a Lipschitz map. Actually, a slightly stronger statement holds:

Proposition 1. Let $X$ be normed, and $F: B_{1}(X) \rightarrow B_{1}(X)$ be $\gamma$-Lipschitz for some $\gamma \in\left\{\alpha, \beta, \chi_{X}\right\}$ with constant $L \leq 1$. If $F$ is uniformly continuous, then $\operatorname{displ}\left(F, B_{1}(X)\right)=0$ and $F$ is not a retraction onto $S_{1}(X)$.

Proof. Since $F$ is uniformly continuous, we can extend $F$ to a continuous map on the completion $\bar{X}$ of $X$. Let $\varepsilon>0$ be given. By Darbo's (or Sadovskiı's) fixed point theorem, the map $(1-\varepsilon) F$ has a fixed point in $B_{1}(\bar{X})$. By continuity, we find some $x \in B_{1}(X)$ in a neighborhood of this point with $\|x-(1-\varepsilon) F(x)\| \leq \varepsilon$; then $\|x-F(x)\| \leq 2 \varepsilon$ and so $\operatorname{displ}(F)=0$. Applying this result to $-F$, we find a sequence $x_{n} \in B_{1}(X)$ such that $y_{n}:=-F\left(x_{n}\right)$ satisfies $\left\|x_{n}-y_{n}\right\| \rightarrow 0$. If $F$ is a retraction onto $S_{1}(X)$, we would have $\left\|F\left(x_{n}\right)-F\left(y_{n}\right)\right\|=\left\|F\left(x_{n}\right)-y_{n}\right\|=2$, contradicting the uniform continuity of $F$

Our main interest is in finding good constants for a large class of spaces. To this end, we modify the construction of [17] carefully. Concerning the minimal displacement problem, we obtain the theoretically best possible constant from (1) (up to an arbitrary small error $\varepsilon>0$ ) in a large class of spaces, and in the class of all normed spaces we obtain this constant up to a factor 2 (and some $\varepsilon>0$ ). Concerning the 
constants for the homotopy and the retraction, we reach by our construction the value $6+\varepsilon$ for each $\gamma$. Moreover, in separable or reflexive spaces the value $4+\varepsilon$ is obtained, and in many spaces even $3+\varepsilon$ (the latter only for $\gamma=\chi_{X}$ ). Since the influence of the radius to our construction is not immediately evident, we do not restrict our attention to the unit sphere.

\section{The abstract construction}

Our main construction works in arbitrary metric spaces.

Definition 2. Given subsets $M$ and $Y$ of a metric space and $\delta_{1}, \delta_{2} \geq 0$, we say that $M$ has a $\left(\delta_{1}, \delta_{2}\right)$-path with respect to $Y$, if there is a sequence of points $e_{n} \in M$ and of (continuous) paths $\Gamma_{n} \subseteq M$ joining $e_{n}$ with $e_{n+1}$ such that the following holds:

1. $\operatorname{dist}\left(\Gamma_{n}, \Gamma_{j}\right)>\delta_{1}$ for $n \geq j+2$.

2. For any $x \in Y$ and any $\varepsilon>0$ we have $\operatorname{dist}\left(x, \Gamma_{n}\right) \geq \delta_{2}-\varepsilon$ for all except at most finitely many numbers $n$.

In this case, we call $\Gamma:=\bigcup_{n} \Gamma_{n}$ a $\left(\delta_{1}, \delta_{2}\right)$-path.

One can always choose $\delta_{2}=\frac{\delta_{1}}{2}$ :

Proposition 2. If $\Gamma$ is a $\left(\delta_{1}, 0\right)$-path for $M$, then $\Gamma$ is also a $\left(\delta_{1}, \frac{\delta_{1}}{2}\right)$-path for $M$ with respect to any $Y$.

Proof. Let $y \in Y$ be given. If $\operatorname{dist}\left(y, \Gamma_{n}\right)>\frac{\delta_{1}}{2}$ for each $n$, we are done. Otherwise, there is some $n$ and some $x \in \Gamma_{n}$ with $d(x, y) \leq \frac{\delta_{1}}{2}$. Then we have for all $k$ with $|k-n| \geq 2$ that $\operatorname{dist}\left(y, \Gamma_{k}\right) \geq \operatorname{dist}\left(x, \Gamma_{k}\right)-d(x, y) \geq \delta_{1}-\frac{\delta_{1}}{2} \geq \frac{\delta_{1}}{2}$

Theorem 1. Let a subset $M$ of a metric space have a $\left(\delta_{1}, \delta_{2}\right)$-path $\Gamma$ with respect to $Y$ (without loss of generality $\left.\delta_{2} \geq \frac{\delta_{1}}{2}\right)$. Then for each $\delta \in\left(0, \frac{\delta_{1}}{2}\right]$ and each $\varepsilon \in(0, \delta)$ there is a continuous map $F: M \rightarrow \Gamma \subseteq M$ with the following properties:

1. $\operatorname{displ}(F, M)>\delta-\varepsilon$.

2. If $A \subseteq M$ is such that $\overline{F(A)}$ is not compact, then $\beta(A) \geq \delta_{1}-2 \delta$ and $\chi_{Y}(A) \geq \delta_{2}-\delta$.

Proof. Let $e_{n}$ and $\Gamma_{n}$ be as in Definition 2. Put

$$
\begin{aligned}
& T_{n}=\left\{x \in M: \operatorname{dist}\left(x, \Gamma_{n}\right) \leq \delta-\varepsilon\right\} \\
& S_{n}=\left\{x \in M: \operatorname{dist}\left(x, \Gamma_{n}\right)<\delta\right\}
\end{aligned}
$$

and $S=\bigcup S_{n}$. Since $\delta \leq \frac{\delta_{1}}{2}$, the set $T_{n}$ intersects $T_{k}$ only if $|k-n| \leq 1$. Moreover, the intersections $I_{n, \pm}=T_{n} \cap T_{n \pm 1}$ contain $e_{n \pm 1}$, and $I_{n,+} \cap I_{n,-}=\emptyset$. By Urysohn's theorem, we find a continuous map $f: T_{n} \rightarrow[n, n+1]$ with $\left.f\right|_{I_{n,-}}=n$ and $\left.f\right|_{I_{n,+}}=$ $n+1$; by the glueing lemma, $f$ is continuous. For $x \notin S$, we put $f(x)=1$, and by Tietze-Urysohn, we extend $f$ inductively to a continuous map $f: \bigcup_{k=1}^{n} \bar{S}_{k} \rightarrow[1, n+1]$. Hence, $f: M \rightarrow[1, \infty)$ is continuous. 
Since $\Gamma_{n}$ are paths, we find a map $g:[1, \infty) \rightarrow \Gamma$ with $g([n, n+1])=\Gamma_{n}, g(n)=e_{n}$, $g(n+1)=e_{n+1}$. We claim that the map $F(x)=g(f(x)+2)$ has the required properties.

For any $x \in M \backslash \bigcup_{n} T_{n}$, the relation $F(x) \in \Gamma$ implies $d(F(x), x)>\delta-\varepsilon$. Moreover, if $x \in T_{n}$, then $f(x) \in[n, n+1]$, and so $F(x) \in \Gamma_{n+2}$ which implies $d(F(x), x)>\delta_{1}-\delta \geq \frac{\delta_{1}}{2} \geq \delta$. Thus, $\operatorname{displ}(F, M) \geq \delta-\varepsilon$.

Now assume that $A \subseteq M$ is such that $\overline{F(A)}$ is not compact. Then $f(A)$ is unbounded, since otherwise $g(\overline{f(A)}+2$ ) is compact (as a continuous image of a compact set) and contains $F(A)$. Hence, we find a sequence $x_{k} \in A$ with $f\left(x_{k}\right)=$ $\alpha_{k} \rightarrow \infty$. By construction, we must have $x_{k} \in S_{n_{k}}$ where $n_{k} \geq \alpha_{k}-1$, and by passing to a subsequence, we may assume that $\left|n_{k}-n_{\ell}\right| \geq 2$ for $k \neq \ell$. In particular, $\left|x_{k}-x_{\ell}\right| \geq \delta_{1}-2 \delta$. Hence, $\beta(A) \geq \delta_{1}-2 \delta$.

Finally, assume that $N \subseteq Y$ is a finite set. Given $\varepsilon_{1}>0$ and $y \in N$, the relation $\operatorname{dist}\left(y, S_{k}\right) \leq \delta_{2}-\delta-\varepsilon_{1}$ holds at most for finitely many numbers $k$. In particular, $d\left(y, x_{k}\right)>\delta_{2}-\delta-\varepsilon_{1}$ for infinitely many $k$. Since this holds for each $y \in N, N$ cannot be a $\left(\delta_{2}-\delta-\varepsilon_{1}\right)$-net, and so $\chi_{Y}(A) \geq \delta_{2}-\delta-\varepsilon_{1}$

For the rest of this paper, let $X$ be a normed space which does not have finite dimension.

Theorem 2. Assume that there is a continuous map $F: B_{r}(X) \rightarrow B_{r}(X)$ with positive minimal displacement $\operatorname{displ}\left(F, B_{r}(X)\right)$. Then:

(i) For each $\varepsilon>0$ there is a homotopy $H: S_{r}(X) \times[0,1] \rightarrow S_{r}(X)$ joining the identity with a constant map such that, for each $\gamma \in\left\{\alpha, \beta, \chi_{X}\right\}$,

$$
\begin{aligned}
\gamma(H(A \times[0,1])) \leq & \frac{2 r}{\operatorname{displ}\left(F, B_{r}(X)\right)} \gamma(A) \\
& +\left(\frac{2 r}{\operatorname{displ}\left(F, B_{r}(X)\right)}-1+\varepsilon\right) \gamma(F(\operatorname{conv}(A \cup\{0\})))
\end{aligned}
$$

for all $A \subseteq S_{r}(X)$.

(ii) For each $\varepsilon>0$ there is a retraction $R$ of $B_{r}(X)$ onto $S_{r}(X)$ such that, for each $\gamma \in\left\{\alpha, \beta, \chi_{X}\right\}$,

$$
\begin{aligned}
\gamma(R(A)) \leq & \frac{2 r}{\operatorname{displ}\left(F, B_{r}(X)\right)}(1+\varepsilon) \gamma(A) \\
& +\left(\frac{2 r}{\operatorname{displ}\left(F, B_{r}(X)\right)}-1+\varepsilon\right) \gamma\left(F\left(B_{r}(X) \cap(1+\varepsilon) \operatorname{conv}(A \cup\{0\})\right)\right)
\end{aligned}
$$

for all $A \subseteq B_{r}(X)$.

Proof. For fixed $c_{1}>0$ and $c_{2} \in(0,1)$ with $0<1-c_{1} c_{2}<\frac{\operatorname{displ}\left(F, B_{r}(X)\right)}{r}$ put

$$
H(x, t)= \begin{cases}r \frac{x-c_{1} t F(x)}{\left\|x-c_{1} t F(x)\right\|} & \text { if } t \leq c_{2} \\ r \frac{\frac{1-t}{1-c_{2}} x-c_{1} c_{2} F\left(\frac{1-t}{1-c_{2}} x\right)}{\left\|\frac{1-t}{1-c_{2}} x-c_{1} c_{2} F\left(\frac{1-t}{1-c_{2}} x\right)\right\|} & \text { if } t \geq c_{2}\end{cases}
$$


and note that

$$
\left\|x-c_{1} t F(x)\right\| \geq r-c_{1} c_{2} r \quad\left(\text { for } t \leq c_{2}\right)
$$

and

$$
\begin{aligned}
\left\|x-c_{1} c_{2} F(x)\right\| & \geq\|x-F(x)\|-\left\|F(x)-c_{1} c_{2} F(x)\right\| \\
& \geq \operatorname{displ}\left(F, B_{r}(X)\right)-\left(1-c_{1} c_{2}\right) r
\end{aligned}
$$

are always positive. Clearly,

$$
\begin{aligned}
& \gamma(H(A \times[0,1])) \\
& \quad \leq r \max \left\{\frac{\gamma(A)+c_{1} \gamma(F(\operatorname{conv}(A \cup\{0\})))}{r\left(1-c_{1} c_{2}\right)}, \frac{\gamma(A)+c_{1} c_{2} \gamma(F(\operatorname{conv}(A \cup\{0\})))}{\operatorname{displ}\left(F, B_{r}(X)\right)-\left(1-c_{1} c_{2}\right) r}\right\}
\end{aligned}
$$

and the minimum of the denominators attains its optimal value

$$
\frac{\operatorname{displ}\left(F, B_{r}(X)\right)}{2} \quad \text { if }\left(1-c_{1} c_{2}\right) r=\frac{\operatorname{displ}\left(F, B_{r}(X)\right)}{2}
$$

i.e. if

$$
c_{1} c_{2}=1-\frac{\operatorname{displ}\left(F, B_{r}(X)\right)}{2 r} .
$$

If we choose $c_{2}$ sufficiently close to 1 , we obtain the claimed estimate. The required retraction is given by

$$
R(x)=H\left(\frac{x}{\|x\|}, \min \{1, k(1-\|x\|)\}\right)
$$

with sufficiently large $k$. Indeed, since $R(x)$ is constant for $\|x\| \leq 1-\frac{1}{k}$, we have

$$
\begin{aligned}
\gamma(R(A)) & =\gamma\left(R\left(A \cap\left\{x:\|x\|>1-\frac{1}{k}\right\}\right)\right) \\
& \leq \gamma\left(H\left(\left(S_{r}(X) \cap \frac{\operatorname{conv}(A \cup\{0\})}{1-\frac{1}{k}}\right) \times[0,1]\right)\right)
\end{aligned}
$$

Thus the theorem is proved

Remark 1. If $\operatorname{displ}\left(F, B_{r}(X)\right)=0$ but $F$ is fixed-point free, then the above constructions (with $c_{1}>1$ and $c_{2}=\frac{1}{c_{1}}$ ) for $H$ and $R$ still work, but one cannot estimate the $\gamma$-Lipschitz constant. These are the constructions mentioned in the introduction. 


\section{Construction for a general normed space}

The following lemma has been proved in [17]:

Lemma 1. If $U \subseteq X$ is separable, complete, and convex, then for each $\varepsilon>0$ there is a retraction $R: X \rightarrow U$ onto $U$ which additionally satisfies $\|x-R(x)\| \leq$ $(1+\varepsilon) \operatorname{dist}(x, U)$.

Using this lemma, we can show now:

Proposition 3. Let $M \subseteq X$ contain a sphere with radius $r>0$. Then, for each $\varepsilon>0, M$ has an $\left(r-\varepsilon, \frac{r-\varepsilon}{2}\right)$-path in this sphere.

Proof. There is no loss of generality to assume that the center of the sphere $S$ is 0 , and it suffices to prove that $M$ has an $\left(r(1+\varepsilon)^{-1}, 0\right)$-path $S$. We choose $e_{1} \in S$ arbitrarily and proceed inductively: Assuming that $e_{1}, \ldots, e_{n} \in S$ and $\Gamma_{1}, \ldots, \Gamma_{n-1} \subseteq S$ are already defined, we let $U_{n}$ denote the linear hull of $e_{1}, \ldots, e_{n}$ and put $U_{0}=\{0\}$. Let $R_{n}$ be a retraction onto $U_{n-1}$ with

$$
\left\|x-R_{n}(x)\right\| \leq\left(1+\varepsilon_{n}\right) \operatorname{dist}\left(x, U_{n-1}\right)
$$

where $\varepsilon_{n}>0$ are chosen such that $r\left(1+\varepsilon_{n}\right)^{-1} \geq r-\varepsilon$. Choose some $f_{n} \notin U_{n}$. Putting

$$
h_{n}(t)=e_{n}+t\left(f_{n}-e_{n}\right) \quad \text { and } \quad u_{n-1}(t)=R\left(h_{n}(t)\right) \quad(0 \leq t \leq 1)
$$

we define the path $\Gamma_{n}$ by

$$
g_{n}(t)=r \frac{h_{n}(t)-u_{n-1}(t)}{\left\|h_{n}(t)-u_{n-1}(t)\right\|}
$$

and put $e_{n+1}=\Gamma_{n}(1)$. Then $\Gamma_{n} \subseteq U_{n+1} \cap S$, and for each $t \in[0,1]$ and each $u \in U_{n-1}$ we have

$$
\begin{aligned}
\left\|g_{n}(t)-u\right\| & =r \frac{\left\|h_{n}(t)-u_{n-1}(t)-r^{-1}\right\| h_{n}(t)-u_{n-1}(t)\|u\|}{\left\|h_{n}(t)-u_{n-1}(t)\right\|} \\
& \geq r \frac{\operatorname{dist}\left(h_{n}(t), U_{n-1}\right)}{\left\|h_{n}(t)-R\left(h_{n}(t)\right)\right\|} \\
& \geq r\left(1+\varepsilon_{n}\right)^{-1} .
\end{aligned}
$$

Hence, for $k \leq n-2$ we have

$$
\operatorname{dist}\left(\Gamma_{n}, \Gamma_{k}\right) \geq \operatorname{dist}\left(\Gamma_{n}, U_{n-1}\right) \geq r\left(1+\varepsilon_{n}\right)^{-1}
$$

and the assertion is proved

It is well-known that for a ball (and thus for a sphere) with radius $r>0$ in $X$ the Kuratowski and Hausdorff measures of non-compactness are always $2 r$ and $r$, respectively (independent of the infinite-dimensional normed space $X$ ). Correspondingly, the value of the corresponding lattice measure of non-compactness belongs to $[r, 2 r]$ (the precise value depends on $X$ ). Using this and recalling that the canonical retraction $\rho$ of $X$ onto a ball never increases any measure of non-compactness (because $\rho(A) \subseteq \overline{\operatorname{conv}}\left(A \cup\left\{x_{0}\right\}\right)$ where $x_{0}$ denotes the center of the ball) we obtain from our previous results the following theorem. 
Theorem 3. Let $M \subseteq X$ contain a sphere $S$ with radius $r>0$. Let $c:=$ $\beta\left(B_{1}(X)\right) \in[1,2]$. Then, for each $\delta \in\left(0, \frac{r}{2}\right)$ and each $\varepsilon>0$, the following existence assertions on maps hold true:

1. There exists a fixed-point free continuous map $F: M \rightarrow S \subseteq M$ with $\operatorname{displ}(F$, $M) \geq \delta-\varepsilon$ such that

$$
\left.\begin{array}{rl}
\chi_{X}(F(A)) & \leq \frac{1}{1-2 \frac{\delta}{r}} \beta(A) \\
\beta(F(A)) & \leq \frac{c}{1-2 \frac{\delta}{r}} \beta(A) \\
\alpha(F(A)) & \leq \frac{2}{1-2 \frac{\delta}{r}} \beta(A)
\end{array}\right\} \quad(A \subseteq M) .
$$

2. There exists a homotopy $H: S \times[0,1] \rightarrow S$ joining the identity with a constant map such that

$$
\chi_{X}(H(A \times[0,1])) \leq(4+1+\varepsilon) \beta(A) \quad(A \subseteq S) .
$$

An analogous statement holds (with a possibly different $H$ ) if one replaces (2) by one of the estimates

$$
\begin{aligned}
& \beta(H(A \times[0,1])) \leq(4+c+\varepsilon) \beta(A) \\
& \gamma(H(A \times[0,1])) \leq(4+2+\varepsilon) \gamma(A)
\end{aligned}
$$

where $\gamma \in\left\{\alpha, \beta, \chi_{X}\right\}$ is arbitrary but fixed.

3. There exists a retraction $R$ of $X$ onto $S$ such that

$$
\chi_{X}(R(A)) \leq(4+1+\varepsilon) \beta(A) \quad(A \subseteq X) .
$$

An analogous statement holds (with a possibly different $R$ ) if one replaces (3) by one of the estimates

$$
\begin{aligned}
& \beta(R(A)) \leq(4+c+\varepsilon) \beta(A) \\
& \gamma(R(A)) \leq(4+2+\varepsilon) \gamma(A)
\end{aligned}
$$

where $\gamma \in\left\{\alpha, \beta, \chi_{X}\right\}$ is arbitrary but fixed.

Proof. The statement for $F$ is a straightforward calculation. Concerning $H$, note that our previous results show that for $0<\varepsilon<\delta<\frac{r}{2}$ a homotopy $H: S \rightarrow S$ joining the identity with a constant map exists with

$$
\gamma(H(A \times[0,1])) \leq \max \left\{\frac{2 r}{\delta-\varepsilon} \gamma(A), \frac{\gamma(S)}{r-2 \delta} \beta(A)\right\} \quad(A \subseteq S) .
$$

For $\gamma=\beta$ and $\varepsilon=0$ (which is not admissible but which we assume by continuity arguments), the optimal choice for $\delta$ concerning the constant in (4) would be the value $\frac{r}{2+\frac{\gamma\left(S_{1}(X)\right)}{2}}$ (which belongs to $\left(0, \frac{r}{2}\right)$ !) for which the constant becomes $\gamma\left(S_{1}(X)\right)+4$; using the estimate $\beta(A) \leq \alpha(A)$ respectively $\chi_{X}(A) \leq \beta(A)$, an analogous calculation works in the cases $\gamma=\alpha$ and $\gamma=\chi_{X}$. For the case $\gamma=\chi_{X}$, we find for $\varepsilon=0$ after the estimate $\beta(A) \leq 2 \chi_{X}(A)$ that the optimal choice for $\delta$ would be $\frac{r}{2+\gamma\left(S_{1}(X)\right)}$ with corresponding constant $2 \gamma\left(S_{1}(X)\right)+4$. The proof concerning $R$ is similar 
The estimates for $F$ imply in particular that for each $\gamma \in\left\{\alpha, \beta, \chi_{X}\right\}$ the estimate

$$
\gamma(F(A)) \leq\left(\frac{1}{2}-\frac{\delta}{r}\right)^{-1} \gamma(A) \quad(A \subseteq M)
$$

holds.

Corollary 1. For each infinite-dimensional normed space $X$, each $L>1$ and each $\varepsilon>0$, there is a continuous function $F: B_{1}(X) \rightarrow S_{1}(X)$ such that

$$
\operatorname{displ}\left(F, B_{1}(X)\right) \geq \frac{1}{2}-\frac{1}{L}-\varepsilon
$$

holds where $F$ is simultaneously $\alpha$-, $\beta$-, and $\gamma$-Lipschitz with constant (at most) $L$.

If we could replace here (i.e. in (5)) the constant $\frac{1}{2}$ by 1 (and could put $\varepsilon=0$ ), this estimate would complement (1). We prove now that one may actually do this in various classes of spaces (at least for $\gamma=\chi_{X}$ ).

\section{Separable or reflexive spaces}

If the underlying space is separable or has a geometry which allows a reduction to the separable case, we obtain sharper results.

Proposition 4. Let $M \subseteq X$ contain a sphere $S$ of radius $r>0$. Then, for each $\varepsilon>0$ and each separable subspace $Y \subseteq X$, the set $M$ has an $(r-\varepsilon, r)$-path in $S \cap Y$ with respect to $Y$.

Proof. The construction of the path is analogous to the construction in the proof of Proposition 3 with the difference that we assume in addition that $\varepsilon_{n} \rightarrow 0$ and that $\operatorname{span}\left\{e_{1}, e_{2}, \ldots\right\}$ is dense in $Y$. The latter can indeed be arranged: By the separability of $Y$ there exist finite-dimensional subspaces $U_{1} \subseteq U_{2} \subseteq \ldots \subseteq Y$ with $Y \subseteq \overline{\bigcup_{n} U_{n}}$ (let, e.g., $U_{n}=\operatorname{span}\left\{y_{1}, \ldots, y_{n}\right\}$ where $\left\{y_{1}, y_{2}, \ldots\right\}$ is dense in $Y$ ). We may assume that $\operatorname{dim} U_{n}=n$, and in the construction of Proposition 3, we choose then $f_{n} \in U_{n+1} \backslash U_{n}$.

To see that $\Gamma$ is an $(r-\varepsilon, r)$-path with respect to $Y$, let $y \in Y$ and $\delta>0$ be given. We find some $N>1$ with $\operatorname{dist}\left(y, U_{N-1}\right)<\delta$ and $r\left(1+\varepsilon_{N}\right)^{-1}>r-\delta$. For all $n \geq N$ our construction implies $\operatorname{dist}\left(\Gamma_{n}, U_{N-1}\right) \geq r\left(1+\varepsilon_{n}\right)^{-1}>r-\delta$. Hence, $\operatorname{dist}\left(y, \Gamma_{n}\right)>r-2 \delta$ for all $n \geq N$

Definition 3. A normed space $X$ has the separable retraction property if there is a separable subspace $Y$ which does not have finite dimension such that for each $\varepsilon>0$ we find a mapping $R: X \rightarrow Y$ which satisfies

$$
\|R(x)-R(y)\| \leq(1+\varepsilon)\|x-y\|+\varepsilon \quad\left(x \in X, y \in B_{1}(Y)\right)
$$

and

$$
\|R(y)-y\| \leq \varepsilon \quad\left(y \in B_{1}(Y)\right) .
$$

Of course, each (infinite-dimensional) separable space has the separable retraction property. More examples have been given in [17]. In particular, if we assume the axiom of choice, then each weakly compactly generated Banach space (and thus each reflexive space) has the separable retraction property. 
Corollary 2. Let $X$ have the separable retraction property, and let $M \subseteq X$ contain a sphere $S$ of radius $r>0$. Then for each $\varepsilon>0$ the set $M$ has an $(r-\varepsilon, r)$ path in $S$ with respect to $X$.

Proof. Let $Y$ be as above, and let $\Gamma$ be an $(r-\varepsilon, r)$-path with respect to $Y$ (Proposition 4). We claim that $\Gamma$ is an $(r-\varepsilon, r)$-path with respect to $X$. Thus, let some $x \in X$ and $\delta>0$ be given. We find a mapping $R: X \rightarrow Y$ which satisfies

$$
\begin{aligned}
\|R(x)-R(y)\| & \leq(1+\delta)\|x-y\|+\delta \\
\| R(y)-y) \| & \leq \delta
\end{aligned}
$$

for all $x \in X$ and all $y \in S \cap Y$. Since $\operatorname{dist}\left(R(x), \Gamma_{n}\right) \geq r-\delta$ for almost all $n$, we have in view of $\Gamma_{n} \subseteq S \cap Y$ that

$$
\begin{aligned}
\operatorname{dist}\left(x, \Gamma_{n}\right) & \geq(1+\delta)^{-1} \operatorname{dist}\left(R(x), R\left(\Gamma_{n}\right)\right)-\delta \\
& \geq(1+\delta)^{-1}\left(\operatorname{dist}\left(R(x), \Gamma_{n}\right)-\delta\right)-\delta \\
& \geq(1+\delta)^{-1}(r-2 \delta)-\delta
\end{aligned}
$$

for almost all $n$. Since the right-hand side is as close to $r$ as we want (for sufficiently small $\delta>0$ ), the claim follows

Together with our previous results, we obtain:

Theorem 4. Let $X$ have the separable retraction property, and put $c=\beta\left(B_{1}(X)\right)$ $\in[1,2]$. Let $M \subseteq X$ contain a sphere $S$ with radius $r>0$. Then, for each $\delta \in\left(0, \frac{r}{2}\right)$, each $\varepsilon>0$, and each $\gamma \in\left\{\alpha, \beta, \chi_{X}\right\}$, the following existence assertions on maps hold true:

1. There exists a fixed-point free continuous map $F: M \rightarrow S \subseteq M$ with $\operatorname{displ}(F$, $\left.B_{r}(X)\right) \geq \delta-\varepsilon$ such that

$$
\left.\begin{array}{rl}
\chi_{X}(F(A)) & \leq \frac{1}{1-\frac{\delta}{r}} \chi_{X}(A) \\
\beta(F(A)) & \leq \frac{c}{1-\frac{\delta}{r}} \chi_{X}(A) \\
\alpha(F(A)) & \leq \frac{2}{1-\frac{\delta}{r}} \chi_{X}(A)
\end{array}\right\} \quad(A \subseteq M) .
$$

2. There exists a homotopy $H: S \times[0,1] \rightarrow S$ joining the identity with a constant map such that

$$
\gamma(H(A \times[0,1])) \leq(4+\varepsilon) \gamma(A) \quad(A \subseteq S)
$$

3. There exists a retraction $R$ of $X$ onto $S$ such that

$$
\gamma(R(A)) \leq(4+\varepsilon) \gamma(A) \quad(A \subseteq X) .
$$

Proof. We use essentially the same calculation as in the proof of Theorem 3. The most remarkable difference is that (4) can be replaced by

$$
\gamma(H(A \times[0,1])) \leq \max \left\{\frac{2 r}{\delta-\varepsilon} \gamma(A), \frac{\gamma(S)}{r-\delta} \chi_{X}(A)\right\} \quad(A \subseteq S)
$$


We estimate $\chi_{X}(A) \leq \gamma(A)$ in (8). For $\varepsilon=0$ the best possible choice for $\delta$ would be $\left(1+\frac{\gamma\left(S_{1}(X)\right)}{2}\right)^{-1} r$ which unfortunately does not belong to $\left(0, \frac{r}{2}\right)$. However, this

calculation shows that $\delta$ should be chosen as large as possible. For $\delta$ close to $\frac{r}{2}$ the estimate in the claim is obtained

Corollary 3. Let the normed space $X$ have the separable retraction property. Then, for each $L>1$ and each $\varepsilon>0$, there exists a continuous function $F: B_{1}(X) \rightarrow$ $S_{1}(X)$ with

$$
\operatorname{displ}\left(F, B_{1}(X)\right) \geq 1-\frac{1}{L}-\varepsilon
$$

where $F$ is $\chi_{X}$-Lipschitz with constant (at most) $L$.

\section{Spaces which contain a copy of $l_{p}$ or $c_{0}$}

By $c_{\text {fin }, p}$ we denote the (incomplete) space of eventually zero sequences, endowed with the $p$-norm. Many spaces contain an isometric copy of some $c_{\text {fin, } p}$, for example Hilbert spaces (put $p=2$ ), $L_{p}(\mu)$ (if it is infinite-dimensional), $C\left([0,1]\right.$ ), or $c_{0}$ (put $p=\infty)$. For such spaces our approach works best.

Lemma 2. Let $X$ contain an isometric copy of $c_{\mathrm{fin}, p}$ for some $p \in[1, \infty]$. Let $M \subseteq X$ contain a sphere $S$ of radius $r>0$. Then $M$ has a $\left(2^{\frac{1}{p}} r-\varepsilon, r\right)$-path $\Gamma \subseteq S$ with respect to $Y:=X$ ( simultaneously for each $\varepsilon>0)$ such that $\operatorname{diam}(\Gamma)=2^{\frac{1}{p}} r$. In particular, $\beta(\Gamma)=\alpha(\Gamma)=2^{\frac{1}{p}} r$.

Proof. We may assume that $X=c_{\text {fin, } p}$ and that $S$ has center 0 . Let $e_{n} \in S$

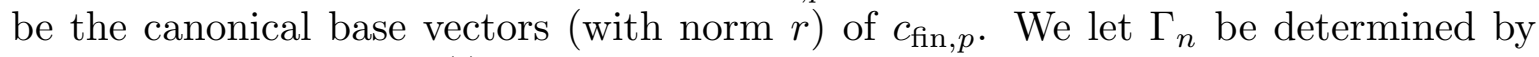
the paths $g_{n}(t)=\frac{r h_{n}(t)}{\left\|h_{n}(t)\right\|}$ in $S$ where $h_{n}(t)=e_{n}+t\left(e_{n+1}-e_{n}\right) \quad(0 \leq t \leq 1)$. For $|k-n| \geq 2$ the vectors $h_{n}(t)$ and $h_{k}(s)$ have disjoint "support" (if we think of $c_{\text {fin, } p}$ as a space of functions $\mathbb{N} \rightarrow \mathbb{R}$ ). Since they have norm $r$, it follows that $\left\|h_{n}(t)-h_{k}(s)\right\|=2^{\frac{1}{p}} r$. Moreover, since the vectors $h_{n}(t)$ and $h_{k}(s)$ correspond to non-negative functions $\mathbb{N} \rightarrow \mathbb{R}$, we have also in the case $|k-n| \leq 1$ the estimate $\left\|h_{n}(t)-h_{k}(s)\right\| \leq 2^{\frac{1}{p}} r$. Hence, $\operatorname{dist}\left(\Gamma_{n}, \Gamma_{k}\right) \geq 2^{\frac{1}{p}} r$ for $\mid k-n \| \geq 2$, and the path $\Gamma=\bigcup_{n} \Gamma_{n}$ satisfies $\operatorname{diam}(\Gamma)=2^{\frac{1}{p}} r$.

Given some $x \in X$ and some $\varepsilon>0$, since $x$ is a null sequence and $g_{n}(t)$ (considered as a function $\mathbb{N} \rightarrow \mathbb{R}$ ) has its "support" at $\{n, n+1\}$ and norm $r$, we have $\operatorname{dist}\left(x, g_{n}(t)\right) \geq r-\varepsilon$ if only $n$ is sufficiently large. Hence, $\operatorname{dist}\left(x, \Gamma_{n}\right) \geq r-\varepsilon$ for almost all $n$

Theorem 5. Let $X$ contain an isometric copy of $c_{\mathrm{fin}, p}$ for some $p \in[1, \infty]$, and put $c=\beta\left(S_{1}(X)\right)$. Let $M \subseteq X$ contain a sphere $S$ of radius $r>0$. Then, for each $\delta \in\left(0,2^{\frac{1}{p}-1} r\right]$ and each $\varepsilon>0$, the following existence assertions on maps hold true:

1. There exists a fixed-point free continuous map $F: M \rightarrow S \subseteq M$ with $\operatorname{displ}(F$, 
$\left.B_{r}(X)\right)>\delta-\varepsilon$ such that

$$
\left.\begin{array}{c}
\chi_{X}(F(A)) \leq \min \left\{\frac{1}{1-\frac{\delta}{r}} \chi_{X}(A), \frac{1+\varepsilon}{2^{\frac{1}{p}}-2 \frac{\delta}{r}} \beta(A)\right\} \\
\alpha(F(A)) \leq \min \left\{\frac{2^{\frac{1}{p}}}{1-\frac{\delta}{r}} \chi_{X}(A), \frac{1+\varepsilon}{1-2^{1-\frac{1}{p}} \frac{\delta}{r}} \beta(A)\right\}
\end{array}\right\}
$$

2. There exists a homotopy $H: S \times[0,1] \rightarrow S$ joining the identity with a constant map such that

$$
\chi_{X}(H(A \times[0,1])) \leq\left(\max \left\{2+1,2^{2-\frac{1}{p}}\right\}+\varepsilon\right) \chi_{X}(A) \quad(A \subseteq S) .
$$

An analogous statement holds (with a possibly different $H$ ) if one replaces (9) by one of the estimates

$$
\begin{aligned}
& \beta(H(A \times[0,1])) \leq\left(\max \left\{2+c, 2^{2-\frac{1}{p}}\right\}+\varepsilon\right) \beta(A) \\
& \alpha(H(A \times[0,1])) \leq(4+\varepsilon) \alpha(A)
\end{aligned}
$$

3. There exists a retraction $R$ of $X$ onto $S$ such that

$$
\chi_{X}(R(A)) \leq\left(\max \left\{2+1,2^{2-\frac{1}{p}}\right\}+\varepsilon\right) \chi_{X}(A) \quad(A \subseteq X) .
$$

An analogous statement holds (with a possibly different $R$ ) if one replaces (10) by one of the estimates

$$
\begin{aligned}
& \beta(R(A)) \leq\left(\max \left\{2+c, 2^{2-\frac{1}{p}}\right\}+\varepsilon\right) \beta(A) \\
& \alpha(R(A)) \leq(4+\varepsilon) \alpha(A)
\end{aligned}
$$

Proof. Since for $F$ as in Theorem 1 corresponding to the previously constructed path $\Gamma$ the inclusion $F(A) \subseteq \Gamma$ holds, we have

$$
\gamma(F(A)) \leq \gamma(\Gamma) \min \left\{\frac{\chi_{X}(A)}{r-\delta}, \frac{\beta(A)}{2^{\frac{1}{p}} r-2 \delta-\varepsilon}\right\} .
$$

For the corresponding homotopy $H$ of Theorem 2 we have (since $\gamma(F(A))=0$ for $\chi_{X}(A) \leq r-\delta$ or $\left.\beta(A)<2^{\frac{1}{p}} r-2 \delta-\varepsilon\right)$ :

$$
\begin{aligned}
\gamma(H(A \times[0,1])) & \leq \min \left\{\max \left\{\frac{2 r}{\delta-\varepsilon} \gamma(A), \min \left\{\frac{\gamma(S) \chi_{X}(A)}{r-\delta}, \frac{\gamma(S) \beta(A)}{2^{\frac{1}{p}} r-2 \delta-\varepsilon}\right\}\right\},\right. \\
& \left.\frac{2 r}{\delta-\varepsilon} \gamma(A)+\left(\frac{2 r}{\delta-\varepsilon}-1\right) \gamma(\Gamma) \min \left\{\frac{\chi_{X}(A)}{r-\delta}, \frac{\beta(A)}{2^{\frac{1}{p}} r-2 \delta-\varepsilon}\right\}\right\} .
\end{aligned}
$$

For $\gamma=\chi_{X}$, we estimate throughout $\beta(A) \leq 2 \chi_{X}(A)$ and find the bound $C \chi_{X}(A)$ where

$$
C=\min \left\{\max \left\{C_{0}, C_{1}\right\}, C_{2}\right\}
$$


with

$$
C_{0}=\frac{2 r}{\delta-\varepsilon}, \quad C_{1}=\frac{1}{1-\frac{\delta}{r}}, \quad C_{2}=C_{0}+\left(C_{0}-1\right) C_{1}
$$

In the case $2^{\frac{1}{p}-1} \geq \frac{2}{3}$ the optimal choice concerning $\max \left\{C_{0}, C_{1}\right\}$ would be $\delta=\frac{2}{3} r$ and $\varepsilon=0$ in which case $C_{0}=C_{1}=3$. In the case $2^{\frac{1}{p}-1}<\frac{2}{3}$ the optimal choice would be $\delta=2^{\frac{1}{p}-1} r$ and $\varepsilon=0$ for which $C_{1} \leq C_{0}=2^{2-\frac{1}{p}} \geq 3$, and we obtain (9). Note that we have in view of $C_{0}-1>1$ always $C_{2} \geq C_{0}+C_{1}$, and so the quantity $C_{2}$ does never improve this estimate.

For $\gamma=\beta$ and $\gamma=\alpha$ we introduce the shortcut $\gamma_{0}=\gamma\left(S_{1}(X)\right)$ and estimate throughout $\chi_{X}(A), \beta(A) \leq \gamma(A)$ to find the bound $D \gamma(A)$ where

$$
D=\min \left\{\max \left\{C_{0}, D_{1}\right\}, \max \left\{C_{0}, D_{2}\right\}, D_{3}\right\}
$$

with

$$
D_{1}=\frac{\gamma_{0}}{1-\frac{\delta}{r}}, \quad D_{2}=\frac{\gamma_{0}}{2^{\frac{1}{p}}-2 \frac{\delta}{r}-\varepsilon}, \quad D_{3}=C_{0}+\left(C_{0}-1\right) \min \left\{D_{1}, D_{2}\right\} \frac{2^{\frac{1}{p}}}{\gamma_{0}}
$$

Similarly, a straightforward calculation shows that the quantity $\max \left\{C_{0}, D_{1}\right\}$ attains its minimal value for $\delta \in\left(0,2^{\frac{1}{p}-1}\right]$ for the choice $\delta=\min \left\{\left(1+\frac{\gamma_{0}}{2}\right)^{-1} r, 2^{\frac{1}{p}-1} r\right\}$ in which case $D_{1} \leq C_{0}=\max \left\{2+\gamma_{0}, 2^{2-\frac{1}{p}}\right\}$. Moreover, $\max \left\{C_{0}, D_{2}\right\}$ attains for the choice $\delta=2^{\frac{1}{p}}\left(\gamma_{0}+2\right) r$ (in $\left.\left(0,2^{\frac{1}{p}-1} r\right)\right)$ and $\varepsilon=0$ its minimal value $\left(4+\gamma_{0}\right) 2^{-\frac{1}{p}}$ which is a worse estimate than $2^{2-\frac{1}{p}}$. The proof concerning $R$ is similar.

Theorems 4 and 5 contain the following complement of (1) for a large class of spaces.

Corollary 4. Let the normed space $X$ have the separable retraction property or let it contain an isometric copy of $c_{\mathrm{fin}, p}(1 \leq p \leq \infty)$. Then, for each $L \in$ $(1,2)$ (respectively even for each $\left.L \in\left(1,\left(1-2^{\frac{1}{p}-1}\right)^{-1}\right]\right)$ and each $\varepsilon>0$, there exists a continuous function $F: B_{1}(X) \rightarrow S_{1}(X)$ with

$$
\operatorname{displ}\left(F, B_{1}(X)\right) \geq 1-\frac{1}{L}-\varepsilon
$$

where $F$ is $\chi_{X}$-Lipschitz with constant (at most) L. Moreover, if $X$ contains an isometric copy of $c_{\mathrm{fin}, \infty}$, it may be arranged that $F$ is also simultaneously $\alpha$-Lipschitz and $\beta$-Lipschitz with constant (at most) L. 


\section{References}

[1] Akhmerov, R. R., Kamenskiı̌, M. I., Potapov, A. S., Rodkina, A. E. and B. N. Sadovskiǔ: Measures of Noncompactness and Condensing Operators. Basel - Boston - Berlin: Birkhäuser Verlag 1992.

[2] Ayerbe Toledano, J. M., Domínguez Benavides, T. and G. López Acedo: Measures of Noncompactness in Metric Fixed Point Theory. Basel - Boston - Berlin: Birkhäuser Verlag 1997.

[3] Benyamini, Y. and Y. Sternfeld: Spheres in infinite-dimensional normed spaces are Lipschitz contractible. Proc. Amer. Math. Soc. 38 (1983), 439 - 445.

[4] Darbo, G.: Punti uniti in trasformazioni a codominio non compatto. Rend. Sem. Mat. Univ. Padova 24 (1955), 84 - 92.

[5] Dugundji, J.: An extension of Tietze's theorem. Pacific J. Math. 1 (1951), 353 - 367.

[6] Franchetti, C.: Lipschitz maps on the unit ball of normed spaces. Confer. Sem. Mat. Univ. Bari 202 (1985)2, pp. 1 - 11.

[7] Franchetti, C.: Lipschitz maps and the geometry of the unit ball in normed spaces. Arch. Math. (Basel) 46 (1986), $76-84$.

[8] Furi, M. and M. Martelli: On the minimal displacement of points under $\alpha$-Lipschitz maps in normed spaces. Boll. Un. Mat. Ital. 9 (1974), $791-799$.

[9] Goebel, K. and W. A. Kirk: Topics in Metric Fixed Point Theory. Cambridge: Cambridge Univ. Press 1990.

[10] Klee, V. L., J.: Some topological properties of convex sets. Trans. Amer. Math. Soc. 78 (1955), $30-45$.

[11] Lin, P. K. and Y. Sternfeld: Convex sets with the Lipschitz fixed point property are compact. Proc. Amer. Math. Soc. 93 (1985), 633 - 639.

[12] Nowak, B.: On the Lipschitzean retraction of the unit ball in infinite dimensional Banach spaces onto its boundary. Bull. Acad. Polon. Sci., Sér. Sci. Math. 27 (1979), $861-864$.

[13] Reich, S.: The fixed point property for nonexpansive mappings I. Amer. Math. Monthly 83 (1976), $266-268$.

[14] Reich, S.: A minimal displacement problem. Comment. Math. Univ. St. Paul. 26 (1978), $131-135$.

[15] Reich, S.: The fixed point property for nonexpansive mappings II. Amer. Math. Monthly 87 (1980), $292-294$.

[16] Sadovskiŭ, B. N.: Limit-compact and condensing operators (in Russian). Uspekhi Mat. Nauk 27 (1972)1, 81 - 146; Engl. transl. in: Russian Math. Surveys 27 (1972)1, 85 155.

[17] Väth, M.: Fixed point free maps of a closed ball with small measures of noncompactness. Collect. Math. 52 (2001), $101-116$.

[18] Wośko, J.: An example related to the retraction problem. Ann. Univ. Mariae CurieSkłodowska (Sect. A) 45 (1991), 127 - 130.

Received 02.05.2002 